\title{
Patient Reported Outcomes in allergic diseases: findings and implications
}

\author{
${ }^{1}$ Respiratory Unit for Continuity of Care, IRCCS Ospedale Policlinico San Martino, \\ Department of Internal Medicine (DiMI), University of Genoa, Genoa, Italy \\ ${ }^{2}$ Personalized Medicine, Asthma \& Allergy, IRCCS Humanitas Research Hospital, Rozzano, Milan, Italy
}

\section{Doi}

10.23822/EurAnnACI.1764-1489.232

Access to the patient's perspective gives a unique key to enhances the range of outcomes that can be evaluated beyond the traditional clinical, biological and functional measures. Patient Reported Outcomes (PROs)-any report directly provided by patients on their experience with health and treatments-are now recognised as an essential part of clinical management and research studies (1). They assess a range of outcomes such as Health Related Quality of Life (HRQoL), level of control, satisfaction, awareness, disease perception. The role of PROs gets more significance when interventions have similar effects in terms of efficacy and safety, and is crucial in any management paradigm that relies on personalized medicine. Moreover, regulatory agencies encourage the inclusion of PROs in the process for approval of new drugs and treatments (1).

Over the last two decades, PROs have emerged as a critical tool for assessing disease impact, level of control and response to treatment in allergic patients. Specifically, the integration of PROs in the healthcare continuum from research to clinical practice, has supplied deep insights into care and management of allergic diseases.

With great interest we read the articles of Dortas Junior (2), El-Qutob (3), and Leiria-Pinto (4), published in this issue of European Annals of Allergy and Clinical Immunology. Each of them developes one of the three steps of the FDA Roadmap to Patient-Focus Outcome Measurement (5) (figure 1).

The observational study by Dortas Junior $e t$ al. (2) is the firsttime evaluation of spirituality in patients with chronic urticaria (CU), controbuiting to better understand the disease (figure 1, step 1). During the last decade, the role of this PRO in chronic dieseases has received more attention, with growing evidence that this dimension of subjective experience is related to physical and psychological wellbeing and contributes to the health outcomes (6). In a study sample of 100 patients, Dortas Junior and co-workers, by mean of validate questionnaires, evaluated the spiritual wellbeing in CU patients with different levels of disease control, and the realtionship between spiritual wellbeing and HRQoL. In their analysis the authors highlight how subjects with poorly controlled CU had significantly lower spiritual wellbeing and worse HRQoL than subjects with controlled CU. Moreover, higher levels of spiritual wellbeing were associated to better HRQoL scores. This study suggests that spirituality may play a role in the experience of CU patients. Additional research is needed to better investigate this construct, and therefore planning targeted intervention programs designed to manage the spiritual well-being of patients with CU.

El-Qutob and co-workers (3) provide the results of the first study that explores the effectiveness of specific immunotherapy based on the combination of allergens in polysensitized patients with asthma and/or rhinitis (figure 1, step 2). Disease control and HRQoL were retrospectively evaluated by mean of validated tools in 39 patients observed in clinical practice. The authors found that the administration of the combination vaccine significantly increases the level of asthma control and HRQoL, and maintains the improvment at 12 months. Although preliminary, these findings are important because highlight for the first time how patients experience specific benefits and changes during the first year of treatment whith immunotherapy based on the combination of allergens.

The availability of simple, psychometrically sound questionnaires should help to implement the assessement of PROS in daily practice. To be used in languages different from the original one, PROS measures must be cross-culturally adapted and and their psychometric characteristics must be verified, following well-established procedures. The Test for Respiratory and Asthma Control in Kids (TRACK) is the only available tool for the assessment of respiratory control in children younger than five years. It was originally developed and validated in English, and then cross-culturally validated into other languages. The article 
Figure 1 - Use of the PROs suggested by FDA Roadmap to Patient-Focus Outcome Measurement.

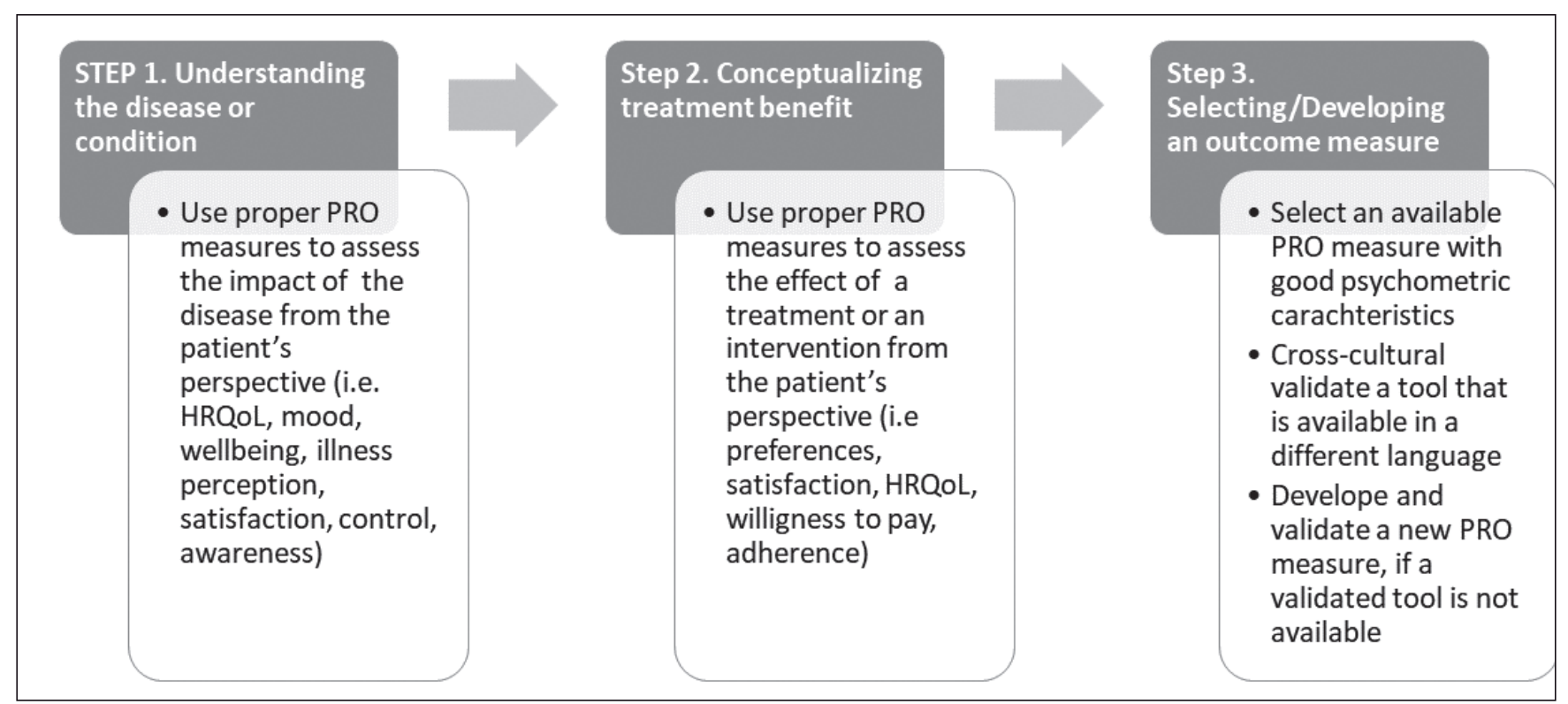

of Pinto et al. (4) describes the results of the rigorous procedure they followed to adaptat and validate the TRACK into the Portuguese language (figure 1, step 3). First, the proper forward-backward translation methodology was adopted to obtain a conceptual and linguistic version that is equivalent to the original one. Second, internal consistency, criterion validity, costruct validity, test-retest reliability, and responsiveness were determined. The results of the validation analyses show that the Portuguese version of TRACK has satisfactory psychometric properties for the assessment of respiratory control in pre-schhol children.

Taken together, these studies offer innovative contributions, all of which provide insights into how researches can capture the disease experience of allergic patients and ensure a comprehensive assessment of disease and drugs impact.

\section{References}

1. Guidance for industry: patient-reported outcome measures: use in medical product development to support labeling claims: draft guidance, Health Qual Life Outcomes 2006; 4:79.
2. Dortas Junior SD, Azizi G, Moret R, et al. Spiritual well-being and quality of life are impaired in chronic urticaria. Eur Ann Allergy Clin Immunol 2021;53(5):221-227. Printed version: Eur Ann Allergy Clin Immunol 2021;53(5):217-223.

3. El-Qutob D, Raducan I, Mencia G. A preliminary study to investigate effectiveness of a mixed extract of Dermatophagoides sp. house dust mites and Alternaria sp. Mold. Eur Ann Allergy Clin Immunol 2021;53(5):234-239. Printed version: Eur Ann Allergy Clin Immunol 2021;53(5):230-235.

4. Leiria-Pinto P, Marques J, Finelli E, et al. Cross-cultural validation of the Portuguese from Portugal version of the Test for Respiratory and Asthma Control in Kids (TRACK) questionnaire. Eur Ann Allergy Clin Immunol 2021;53(5):214-220. Printed version: Eur Ann Allergy Clin Immunol 2021;53(5):210-216.

5. Available from https://www.fda.gov/Drugs/DevelopmentApprovalProcess/DrugDevelopmentToolsQualificationProgram/ ucm 284077.htm.

6. Oman D. Why Religion and Spirituality Matter for Public Health: Evidence, Implications, and Resources. Springer International Publishing, Cham, Switzerland 2018. 